\title{
Resistance to Plum pox virus Strain C in Arabidopsis thaliana and Chenopodium foetidum Involves Genome-Linked Viral Protein and Other Viral Determinants and Might Depend on Compatibility With Host Translation Initiation Factors
}

\author{
María Calvo, Sandra Martínez-Turiño, and Juan Antonio García \\ Departamento de Genética Molecular de Plantas, Centro Nacional de Biotecnología (CNB-CSIC), Campus Universidad \\ Autónoma de Madrid, 28049 Madrid, Spain
}

Submitted 13 May 2014. Accepted 16 July 2014.

\begin{abstract}
Research performed on model herbaceous hosts has been useful to unravel the molecular mechanisms that control viral infections. The most common Plum pox virus (PPV) strains are able to infect Nicotiana species as well as Chenopodium and Arabidopsis species. However, isolates belonging to strain C (PPV-C) that have been adapted to Nicotiana spp. are not infectious either in Chenopodium foetidum or in Arabidopsis thaliana. In order to determine the mechanism underlying this interesting host-specific behavior, we have constructed chimerical clones derived from Nicotiana-adapted PPV isolates from the D and C strains, which differ in their capacity to infect $A$. thaliana and $C$. foetidum. With this approach, we have identified the nuclear inclusion a protein (VPg+Pro) as the major pathogenicity determinant that conditions resistance in the presence of additional secondary determinants, different for each host. Genome-linked viral protein (VPg) mutations similar to those involved in the breakdown of eIF4E-mediated resistance to other potyviruses allow some PPV chimeras to infect $A$. thaliana. These results point to defective interactions between a translation initiation factor and the viral VPg as the most probable cause of host-specific incompatibility, in which other viral factors also participate, and suggest that complex interactions between multiple viral proteins and translation initiation factors not only define resistance to potyviruses in particular varieties of susceptible hosts but also contribute to establish nonhost resistance.
\end{abstract}

Plum pox virus (PPV) belongs to the genus Potyvirus, one of the largest groups among picorna-like plant viruses (Ivanov et al. 2014; López-Moya et al. 2009). It has a positive singlestranded RNA genome of approximately $10 \mathrm{~Kb}$ covalently

Current address for M. Calvo: Centogene AG, Schillingallee 68, 18057 Rostock, Germany.

Corresponding authors: J. A. García; E-mail: jagarcia@cnb.csic.es; and M. Calvo; E-mail: mariacalvo83@gmail.com

* The $\boldsymbol{e}$-Xtra logo stands for "electronic extra" and indicates that two supplementary figures, supplementary text, and a supplementary table are published online and that Figures 3, 5, and 6 appear in color online.

(C) 2014 The American Phytopathological Society fused to the genome-linked viral protein (VPg). The genomic RNA is translated into a large polyprotein and a smaller frameshift product that are cleaved by three self-encoded proteases, i.e., P1, HCpro, and the Pro domain of the nuclear inclusion a (NIa) protein, thus generating at least eleven mature viral products (García et al. 2014; Šubr and Glasa 2013).

Although PPV is mostly known for being the causal agent of the devastating sharka disease in Prunus species, it has a wide host range that includes not only the aforementioned woody species but also herbaceous hosts. Herbaceous species are not generally considered to be important reservoirs for PPV in nature (Llácer 2006) but can be used as experimental hosts for the study of PPV in the laboratory, due to their high susceptibility towards infection, easy manipulation, and the availability of genetic tools that enable the identification of plant-virus interaction factors. For instance, species such as Nicotiana benthamiana and $N$. clevelandii, which are widely used hosts in plant virology (Goodin et al. 2008; van Dijk et al. 1987), are susceptible to all PPV strains. Species from the genus Chenopodium have traditionally been used as indicator plants, due to the triggering of a hypersensitive response (HR) that results in the development of local lesions upon inoculation of many viruses, including potyviruses (Cooper 2001; Yongqiang et al. 2012). In the particular case of PPV, the different isolates were initially classified according to the virulence of the lesions that they produced in this host (Sutic et al. 1971). More recently, Arabidopsis thaliana has also become an important host due to the high number of available genetic resources that greatly facilitate studies on plant-virus interactions (Decroocq et al. 2006; Elena et al. 2011; Ouibrahim and Caranta 2013).

Although the most prevalent PPV strains, Marcus (M), Dideron (D), and Recombinant (Rec), are able to infect $N$. clevelandii, C. foetidum, and A. thaliana, PPV isolates belonging to the Cherry (C) strain (PPV-C), which are highly infectious in Nicotiana spp., are not infectious in C. foetidum and in usual $A$. thaliana accessions such as Col-0 and Ler (Calvo et al. 2014; Crescenzi et al. 1997; Decroocq et al. 2006; Fanigliulo et al. 2003).

So far, the mechanisms that prevent the infection of PPV-C in $C$. foetidum and $A$. thaliana remain mostly unknown. In the case of $A$. thaliana, the mechanism that impedes viral initial replication of the Nicotiana-adapted PPV-C SoC isolate (PPVSoC) has been related to resistance $(R)$ gene-mediated resistance pathways (Decroocq et al. 2006). In the case of $C$. foe- 
tidum, it is possible that this host develops effector triggered immunity (Moffett 2009) against PPV that culminates in HR in response to the usual PPV isolates and in extreme resistance (ER), with no visible phenotype, upon inoculation of PPV-C isolates. Another possibility is that the infectivity failure of PPV-C may be due to the nonestablishment of plant-virus interactions that might be needed for an efficient viral replication. In any case, the viral factors from PPV-C that are involved in these putative processes have not been identified. We have taken advantage of infectious full-length cDNA clones from Nicotiana-adapted PPV isolates belonging to strains D (pICPPV-R based on the Rankovic isolate) and C (pICPPVSwCM based on the SwCMp isolate) (Calvo et al. 2014; López-Moya and García 2000) to map the genetic determinants conditioning resistance in these hosts. Our findings let us speculate on the mechanism that most probably operates in both A. thaliana and C. foetidum.

\section{RESULTS}

PPV-SwCM does not infect $A$. thaliana or $C$. foetidum.

Taking advantage of the high infectivity of the full-length cDNA clone pICPPV-SwCM, derived from the PPV-C isolate PPV-SwCMp, in Nicotiana spp. (Calvo et al. 2014), the viral progeny (hereafter called PPV-SwCM) from systemically infected $N$. clevelandii leaves was used as inoculum in the following infectivity tests that were performed in A. thaliana and $C$. foetidum. Based on the results obtained by Decroocq and associates (2006) with the Nicotiana-adapted PPV-C SoC isolate, A. thaliana (Ler) wild-type plants and mutants $\operatorname{rarl-10}$ and sgt $1 b$ - 1/rar1-10 were challenged with PPV-SwCM to test whether the virus was able to replicate in inoculated leaves of these mutants, which are defective in $R$ gene-triggered resistance (Austin et al. 2002; Azevedo et al. 2002). No local or systemic symptoms of infection were observed and no viral accumulation was detected, either by immunoblot analysis or by immunocapture reverse transcription-polymerase chain reaction (IC-RT-PCR) in any of the plants (Supplementary Fig. S1A and B).

In the case of $C$. foetidum, the absence of local lesions upon inoculation of PPV-SwCM correlated with the lack of viral accumulation assessed by immunoblot analysis at 10 days postinoculation (dpi). Therefore, in the following experiments, the infectivity in this species was evaluated just by observation of local lesions.

\section{PPV-SwCM and PPV-R do not affect the infectivity of each other in A. thaliana or C. foetidum.}

To test whether a putative resistance mechanism preventing the infection of PPV-SwCM could also act against a virus different from the one triggering this response or if a virulent virus could enable the infectivity of PPV-SwCM, the viral progeny of the pICPPV-R cDNA clone (hereafter PPV-R) obtained from $N$. clevelandii-infected leaves was co-inoculated with PPV-SwCM in A. thaliana Ler plants.

For this purpose, PPV-SwCM- and PPV-R-infected $N$. clevelandii extracts were mixed 50:50 and 90:10 (SwCM:R). As positive controls, PPV-R and healthy plant extracts were mixed in the same ratios (healthy:R). At 9 dpi, local symptoms typical for PPV-R infection were observed in the co-inoculated rosette leaves of plants challenged with both SwCM:R or
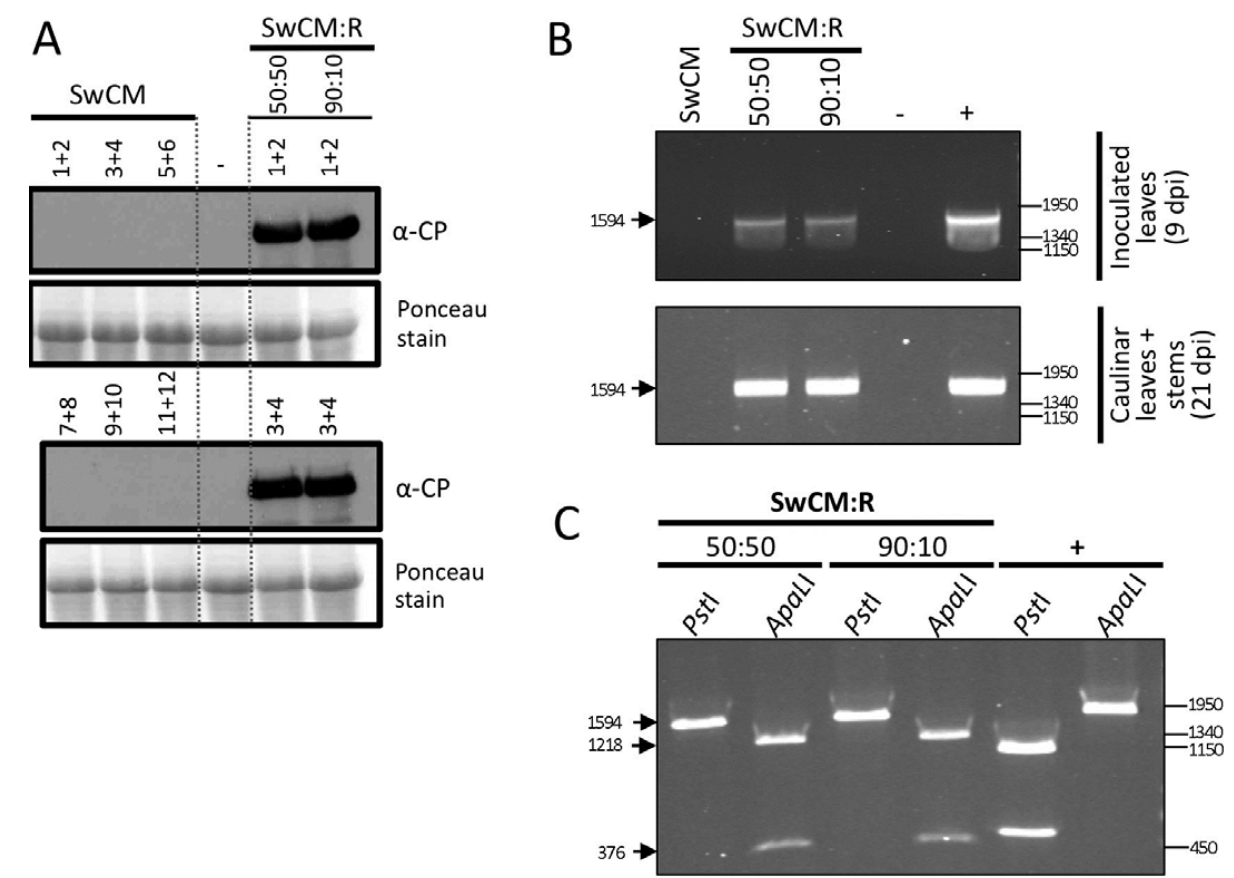

Fig. 1. Infectivity of mixed Plum pox virus (PPV)-SwCM:PPV-R (SwCM:R) inocula in Arabidopsis thaliana. A, Viral accumulation assessed by anti-coat protein immunoblot analysis (serum dilution 1:100,000) at 9 days postinoculation in inoculated rosette leaves of plants of $A$. thaliana challenged either with extract of PPV-SwCM-infected Nicotiana clevelandii leaves (SwCM) or with a mixture of extracts of PPV-SwCM- and PPV-R-infected N. clevelandii leaves (SwCM:R) in 50:50 or 90:10 proportions. Samples from 12 plants inoculated with PPV-SwCM and samples from four plants inoculated with SwCM:R in 50:50 or 90:10 proportions, were analyzed in pairs. A sample from healthy A. thaliana was used as a negative control (-). Membrane stained with Ponceau red showing the Rubisco is included as a loading control in the lower panels. B, Immunocapture reverse transcription-polymerase chain reaction (IC-RT-PCR) amplification of a viral cDNA fragment (nucleotides 8,196 to 9,795 of PPV-SwCM and 8,193 to 9,786 of PPV-R) from samples of SwCMand SwCM:R-inoculated plants (upper panel, inoculated rosette leaves; lower panel, stems and caulinar noninoculated leaves). A sample from healthy A. thaliana was used as a negative control (-). A SwCM band amplified from a sample from infected $N$. clevelandii was used as a positive control (+). C, Restriction fragment length polymorphism analysis in which the IC-RT-PCR-amplified fragments were digested with PstI and ApaLI, which specifically cut PPV-SwCM- and PPV-R-amplified sequences, respectively. The digestion with PstI of a SwCM-amplified fragment from a sample from infected $N$. clevelandii was used as a positive control (+). The expected size of the amplified (B and C) or digested (C) fragments is indicated with black arrows on the left side of the panels. Molecular weight marker sizes are indicated on the right side of the panels. 
healthy:R mixed extracts. Symptoms consisted of isolated local lesions that could sometimes be difficult to observe due to inoculation damage. There was a positive correlation between symptom appearance and viral accumulation when assessed by immunoblot analysis (Fig. 1A) and IC-RT-PCR (Fig. 1B).

At 21 dpi, plants co-inoculated with SwCM:R and healthy:R (both 50:50 and 90:10) mixed extracts showed strong chlorosis on caulinar leaves coupled with plant growth impairment. Samples from stems and caulinar leaves were collected together and were analyzed by IC-RT-PCR. There was, again, a positive correlation between symptom appearance and viral amplification (Fig. 1B). IC-RT-PCR products were subjected to a restriction fragment length polymorphism (RFLP) analysis using diagnostic enzymes PstI and ApaLI, which were able to specifically cut in the PPV-SwCM- or PPV-R-amplified sequences, respectively, in order to determine which viruses were actually infecting these plants (Fig. 1C). The fragments amplified from plants that had been co-inoculated with the mixed SwCM:R extracts were fully cut with ApaLI and not with PstI, which indicated that the infecting virus was PPV-R in all cases and no detectable PPV-SwCM amplification had taken place.
Thus, the presence of PPV-SwCM in the inocula did not prevent the infection of PPV-R and there was no complementation between the two viruses enabling the infection of PPV-SwCM.

In the case of $C$. foetidum, plants were divided in three groups and were initially inoculated with either PPV-R or SwCM or were mock-inoculated (Table 1). As expected, at $8 \mathrm{dpi}$, local lesions were visible in plants inoculated with PPV-R (group 1), and no lesions were observed in PPV-SwCM- or mock-inoculated plants (groups 2 and 3) (Table 1). At this time, plants from groups 2 and 3 were reinoculated in the same and in upper leaves with PPV-R. Plants from group 1, which had already developed local lesions, were only inoculated in upper leaves (Table 1). It was expected that if PPV-SwCM were triggering a local or systemic defense response, PPV-R would not be able to cause local lesions on plants that had been previously inoculated with PPV-SwCM, while there would be no effect on the initially mock- or PPV-R-inoculated plants. Eight days after the second inoculation, the appearance of local lesions was again evaluated. The result of this experiment revealed that the initial inoculation of PPV-SwCM had no local or systemic effect on the subsequent infectivity of PPV-R, since a similar number of local lesions, which appeared at the same time and developed in the same

Table 1. Effect of Plum pox virus (PPV) SwCM on the infectivity of PPV-R in Chenopodium foetidum ${ }^{\mathrm{a}}$

\begin{tabular}{|c|c|c|c|c|c|c|}
\hline \multirow[b]{2}{*}{ Timepoint } & \multicolumn{2}{|c|}{ Group 1} & \multicolumn{2}{|l|}{ Group 2} & \multicolumn{2}{|c|}{ Group 3} \\
\hline & $\begin{array}{l}\text { Lower leaves: } \\
\text { PPV-R }\end{array}$ & $\begin{array}{l}\text { Upper leaves: }^{\mathrm{c}} \\
\text { PPV-R }\end{array}$ & $\begin{array}{c}\text { Lower leaves: }^{\text {d }} \\
\text { 1) PPV-SwCM 2) PPV-R }\end{array}$ & $\begin{array}{l}\text { Upper leaves: } \\
\text { PPV-R }\end{array}$ & $\begin{array}{l}\text { Lower leaves: }^{\mathrm{d}} \\
\text { 1) Mock 2) PPV-R }\end{array}$ & $\begin{array}{l}\text { Upper leaves: } \\
\text { PPV-R }\end{array}$ \\
\hline $\begin{array}{l}8 \text { dpi after first inoculation } \\
8 \text { dpi after second inoculation }\end{array}$ & $+^{\mathrm{d}}$ & $\ldots$ & $\begin{array}{l}-^{\mathrm{e}} \\
+^{\mathrm{d}}\end{array}$ & $+^{\mathrm{d}}$ & $-\overline{-}$ & 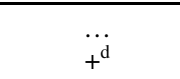 \\
\hline
\end{tabular}

${ }^{a}+$ indicates that local lesions appeared at 8 dpi. Lesions were similar according to number and phenotype. - indicates that no local lesions were observed. Ellipses (...) indicate leaves that were not inoculated.

${ }^{\mathrm{b}}$ Leaves inoculated only at day 0 .

${ }^{\mathrm{c}}$ Leaves that are located above the first inoculated leaves in the plant and were inoculated at day 8 .

${ }^{\mathrm{d}}$ Leaves inoculated two times, first at day 0 and, then, 8 days later.
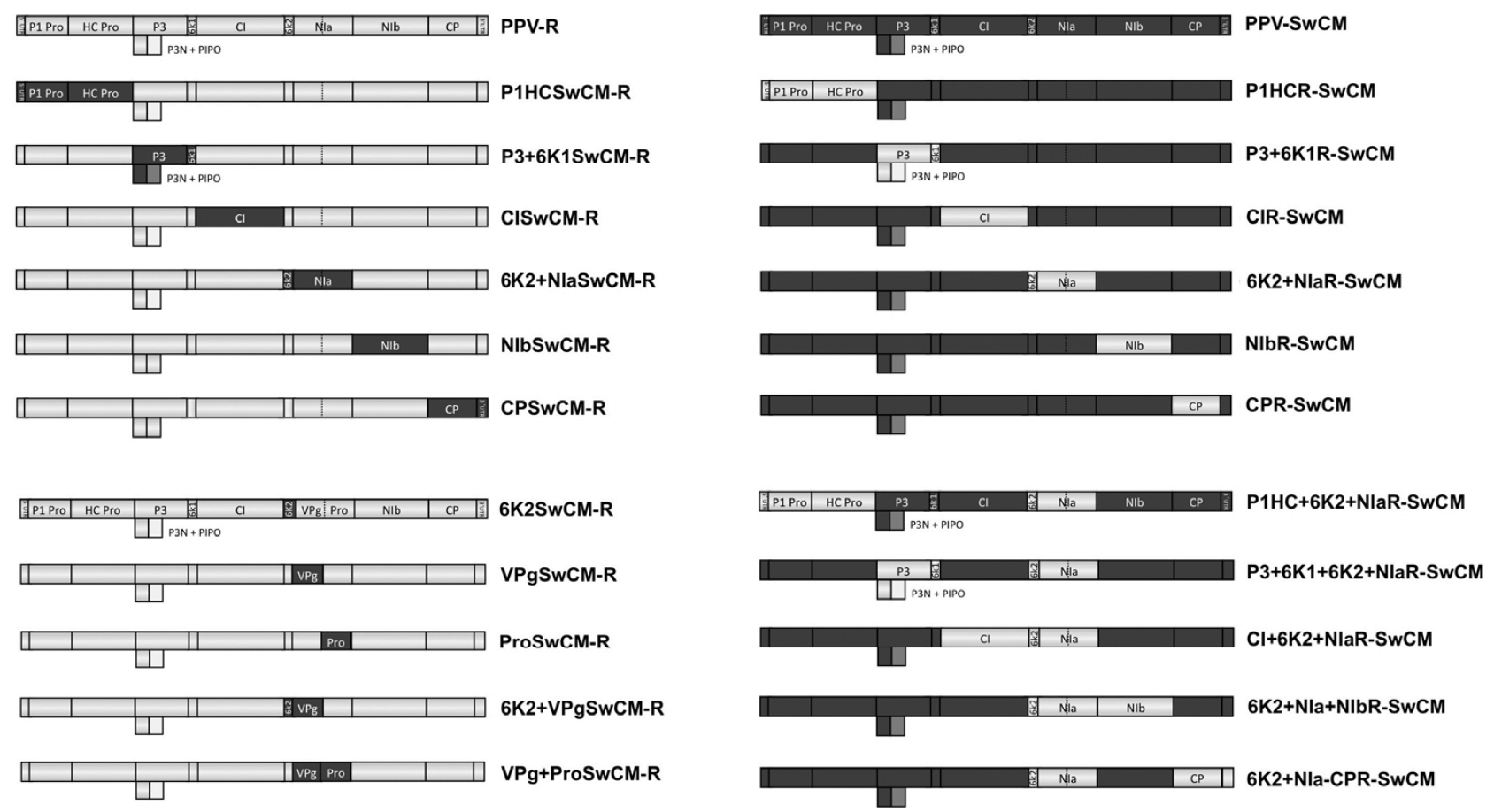

Fig. 2. Schematic representation of full-length cDNA clones pICPPV-R and pICPPV-SwCM and pICPPV chimeras derived from them. Rectangular boxes represent the different coding sequences and the $5^{\prime}$ - and $3^{\prime}$-untranslated regions of the PPV-SwCM (dark gray) and PPV-R (light gray) genome. Intron I from the ST-LSI gene of tomato (interrupting the P3 cistron) has not been shown in order to simplify the schemes. The name of each construct is indicated on the right side. 
sequences outside the $6 \mathrm{~K} 2+\mathrm{NIa}$ region for the corresponding ones of PPV-R does not seem to affect the infectivity of this virus in A. thaliana and C. foetidum.

\section{The VPg and Pro domains of PPV-SwCM NIa have host-specific pathogenicity determinants preventing its infection in A. thaliana and $C$. foetidum.}

In order to do a finer mapping of the coding sequences within the $6 \mathrm{~K} 2+\mathrm{NIa}$ region of PPV-SwCM that were preventing the infectivity of the $6 \mathrm{~K} 2+\mathrm{NIaSwCM}-\mathrm{R}$ chimera in $A$. thaliana and $C$. foetidum, additional constructs separating the $6 \mathrm{~K} 2, \mathrm{VPg}$, and Pro or combining the $6 \mathrm{~K} 2+\mathrm{VPg}$ and the $\mathrm{VPg}+$ Pro coding sequences of PPV-SwCM were assembled in the pICPPV-R backbone (Fig. 2C). Extracts from systemically infected $N$. clevelandii tissue were used to evaluate the infectivity of these new chimerical viruses in both hosts.

Taking together the results of two independent experiments, symptoms on inoculated and caulinar leaves were observed in all $A$. thaliana plants inoculated with the control PPV-R as well as in all plants inoculated with the viral progeny of 6K2SwCM-R (PPV-R containing the 6K2 of PPV-SwCM) and in only some plants inoculated with the viral progeny of ProSwCM-R or $6 \mathrm{~K} 2+\mathrm{VPgSwCM}-\mathrm{R}$ (PPV-R containing the Pro or $6 \mathrm{~K} 2-\mathrm{VPg}$, respectively, of PPV-SwCM), in which symptoms were milder than those caused by PPV-R. No symptoms were observed in plants infected with VPgSwCM-R, VPg+ProSwCM-R, or 6K2+NIaSwCM-R (PPV-R with the VPg, VPgPro, or 6K2-VPg-Pro, respectively, of PPV-SwCM).

Local viral accumulation was detected by immunoblot analysis in leaves inoculated with the viral progeny of all chimeras, including the control $6 \mathrm{~K} 2+\mathrm{NIaSwCM}-\mathrm{R}$, although not in all the analyzed plants. The highest accumulation levels were reached by $6 \mathrm{~K} 2 \mathrm{SwCM}-\mathrm{R}$, which was the only virus of this set of chimeras able to infect all inoculated plants (Fig. 4A).

Sample analysis from caulinar leaves and stems revealed that, as expected, $6 \mathrm{~K} 2+\mathrm{NIaSwCM}-\mathrm{R}$ was not able to cause a systemic infection. Also, no viral accumulation was detected in any sample from plants inoculated with VPg+ProSwCM-R. Viral CP was detected by immunoblot analysis in all samples from plants inoculated with 6K2SwCM-R and in some samples from plants inoculated with VPgSwCM-R, ProSwCM-R, and $6 \mathrm{~K} 2+\mathrm{VPgSwCM}-\mathrm{R}$ (Fig. 4A and $\mathrm{B}$ ).

The genome sequence of the systemic viral progeny from some of the samples (single plants or pools) showing $\mathrm{CP}$ accumulation was analyzed in order to determine whether any mutations had been introduced in the exchanged regions (sequences

Table 3. Infectivity of the viral progeny of $6 \mathrm{~K} 2+\mathrm{NIaR}-\mathrm{SwCM}$-derived chimeras in Chenopodium foetidum

\begin{tabular}{lcccc}
\hline Inoculum $^{\mathbf{a}}$ & Initial tests $^{\mathbf{b}}$ & $\mathbf{A}^{\mathbf{c}}$ & $\mathbf{B}^{\mathbf{c}}$ & Total \\
\hline pICPPV-SwCM & $0 / 26^{\mathrm{d}}$ & $0 / 8$ & $0 / 28$ & $0 / 62$ \\
R-SwCM & & & & \\
6K2+NIa & $\ldots$ & $0 / 8$ & $2 / 24$ & $2 / 32$ \\
P1HCpro+ 6K2+NIa & $\ldots$ & $\ldots$ & $3 / 12$ & $3 / 12$ \\
P3+6K1+6K2+NIa & $\ldots$ & $\ldots$ & $76 / 12$ & $76 / 12$ \\
CI+ 6K2+NIa & $\ldots$ & $\ldots$ & $1 / 12$ & $1 / 12$ \\
6K2+NIa+ NIb & $\ldots$ & $\ldots$ & $0 / 12$ & $0 / 12$ \\
6K2+NIa+ CP & $\ldots$ & $\ldots$ & $2 / 12$ & $2 / 12$ \\
PPV-R & $+++^{\mathrm{e}}$ & +++ & +++ & +++ \\
\hline
\end{tabular}

${ }^{a}$ Viral progeny from Nicotiana clevelandii systemically infected leaf extracts; the PPV-R (Plum pox virus strain R) coding sequences included in the pICPPV-SwCM backbone are indicated.

b Ellipses (...) indicate that the chimeras were not analyzed.

c Independent experiments.

${ }^{d}$ Number of lesions/number of inoculated leaves.

e +++ indicates a large number of lesions which were not accurately evaluated but tended to be at least 40 per leaf. derived from PPV-SwCM), and the results are summarized in Table 4. No mutations were detected in the Pro coding sequence of the viral progeny from any of the four plants infected with ProSwCM-R. However, the viral progeny of all $6 \mathrm{~K} 2+\mathrm{VPg}-$ SwCM-R-infected plants that were analyzed had introduced single mutations P1968S and F2017L (this one appearing in two independent samples) affecting the VPg coding sequence. Interestingly, these mutations were also found in the viral progeny of samples inoculated with VPgSwCM-R, P1968S in two pools of infected plants and F2017L in the third pool analyzed.

To test whether the viral progeny from $A$. thaliana plants infected with ProSwCM-R and 6K2+VPgSwCM-R (these including the aforementioned mutations) showed an increased infectivity, inocula from these infected samples were used to rechallenge $A$. thaliana plants (Fig. 4C). Systemic symptoms of infection were observed only in plants that had been inoculated with progenies of $6 \mathrm{~K} 2+\mathrm{VPgSwCM}-\mathrm{R}$-infected plants (two of three progenies, one of four inoculated plants in each case). Viral accumulation analyses corroborated symptom observation (Fig. 4C). According to sequence analyses, the viral progeny maintained the same amino acid changes and did not introduce additional mutations in the PPV-SwCM-derived region in both infected plants.

The viral progeny from VPgSwCM-R- and 6K2+VPgSwCM-R-infected $A$. thaliana plants, which included mutations P1968S or F2017L (VPgSwCM-R) or mutation F2017L $(6 \mathrm{~K} 2+\mathrm{VPgSwCM}-\mathrm{R})$, were propagated in $N$. clevelandii in order to determine whether VPg mutations selected in A. thaliana affected the ability of the chimera to infect the host in which they were initially propagated. Chimeras carrying the aforementioned mutations systemically infected $N$. clevelandii plants with typical symptoms. IC-RT-PCR analyses showed that, at $21 \mathrm{dpi}$, the PPV-SwCM VPg-derived region had not reverted to the original sequence in either of the mutated chimeras. This result seems to indicate that, whereas wild-type $\mathrm{SwCM} \mathrm{VPg}$ is functional only in $N$. clevelandii plants, VPg

Table 4. Systemic infectivity of the viral progeny of $6 \mathrm{~K} 2+\mathrm{NIaSwCM}-\mathrm{R}-$ derived chimeras in Arabidopsis thaliana

\begin{tabular}{|c|c|c|c|c|}
\hline Inoculum $^{\mathrm{a}}$ & Symptoms $^{b}$ & Infectivity $^{\mathrm{c}}$ & Mutations $^{\mathbf{d}}$ & $\begin{array}{c}\text { Affected } \\
\text { coding } \\
\text { sequence }\end{array}$ \\
\hline pICPPV-R & +++ & $10 / 10$ & $\ldots$ & $\ldots$ \\
\hline \multicolumn{5}{|l|}{ SwCM-R } \\
\hline $6 \mathrm{~K} 2+\mathrm{NIa}$ & - & $0 / 16$ & $\ldots$ & $\ldots$ \\
\hline $6 \mathrm{~K} 2$ & +++ & $8 / 8$ & - & $\ldots$ \\
\hline Pro & + & $5-7 / 16$ & - & $\ldots$ \\
\hline VPg & - & $4-8 / 16$ & $\begin{array}{l}\text { P1968S } \\
(\text { P114S) })^{\mathrm{e}, \mathrm{f}} \\
\text { F2017L }(\mathrm{F} 163 \mathrm{~L})^{\mathrm{e}}\end{array}$ & VPg \\
\hline $6 \mathrm{~K} 2+\mathrm{VPg}$ & + & $3-6 / 16$ & $\begin{array}{l}\text { P1968S (P114S) } \\
\text { F2017 (F163L) }\end{array}$ & VPg \\
\hline $\mathrm{VPg}+\mathrm{Pro}$ & - & $0 / 16$ & $\ldots$ & $\ldots$ \\
\hline
\end{tabular}

${ }^{a}$ Viral progeny from Nicotiana clevelandii systemically infected leaf extracts; the Plum pox virus (PPV)-SwCM coding sequences included in the pICPPV-R backbone are indicated.

b The number of + symbols indicates a semiquantitative estimation of the intensity of the symptoms.

${ }^{c}$ Positive samples from caulinar leaves and stems in coat protein (CP) immunoblot analyses. Since some positive samples had been collected and analyzed in pools, exact numbers cannot be given in all cases.

${ }^{\mathrm{d}}$ Mutations in the exchanged region. Amino acid positions relative to the VPg protein sequence is indicated in brackets. Ellipses (...) indicate that the virus (pICPPV-R) was already adapted to the host or there were no infected plants to make an analysis $(6 \mathrm{~K} 2+\mathrm{NIa}$ and $\mathrm{VPg}+\mathrm{Pro}) ;-$ means that no symptoms or no mutations were observed.

e Samples analyzed in pools of two plants.

${ }^{\mathrm{f}}$ Mutation appearing in two pools of plants, and therefore, present in at least two independent plants. 
variants P1968S and F2017L are functional in both hosts, $N$. clevelandii and A. thaliana, although the possibility of compensatory mutations being introduced in other regions of the viral genome cannot be discarded.

In $C$. foetidum, the viral progeny of the $6 \mathrm{~K} 2+\mathrm{NIaSwCM}-\mathrm{R}$ chimera was able to cause local lesions in only a few inoculated leaves and in a very low number. 6K2SwCM-R was able to cause lesions that were similar in number and morphology to those of PPV-R (Fig. 5), while lesions produced by ProSwCM-R appeared to be less numerous and milder at 8 dpi (Fig. 5), even though they eventually acquired the typical necrotic phenotype. The chimeras that contained the VPg of SwCM, alone or combined with the $6 \mathrm{~K} 2$ or Pro coding sequences, did not cause detectable local lesions (Fig. 5).

ProSwCM-R progeny from $C$. foetidum lesions was amplified by a passage in $N$. clevelandii plants and reinoculated in new $C$. foetidum plants. At $8 \mathrm{dpi}$, the same peculiar local lesion phenotype as in the first inoculation was observed (data not shown). Thus, it seems to be an inherent trait of this chimera.

All together, these results indicate that the presence of the PPV-SwCM NIa (VPg+Pro) coding sequence causes a drastic drop of PPV infectivity in A. thaliana and C. foetidum, although VPg and Pro separately also have a significant contribution.

\section{Different combinations of the 6K2+NIa and other coding sequences of PPV-R are necessary for PPV-SwCM to gain infectivity \\ in A. thaliana and C. foetidum.}

According to the previous results, the replacement of $6 \mathrm{~K} 2+\mathrm{NIa}$ or any other coding sequences of PPV-SwCM by the corresponding ones of PPV-R in the pICPPV-SwCM backbone does not cause an important gain of infectivity, either in $A$ thaliana or in C. foetidum (Table 2; Fig. 3), even though the reciprocal exchange (6K2+NIa coding sequence of PPV-SwCM in the pICPPV-R backbone) drastically reduced the infectivity of the resulting chimera $(6 \mathrm{~K} 2+\mathrm{NIaSwCM}-\mathrm{R})$, as compared with PPV-R. Therefore we hypothesized that other viral coding regions, necessarily combined with $6 \mathrm{~K} 2+\mathrm{NIa}$, could also be contributing to the inability of PPV-SwCM to establish an infection in these hosts, despite the fact that the exchange of any of the other coding sequences separately did not affect the infectivity of PPV-R (Table 2).

Thus, additional chimeras that combined P1-HCpro, P3+6K1, $\mathrm{CI}, \mathrm{NIb}$, and $\mathrm{CP}$ with $6 \mathrm{~K} 2+\mathrm{NIa}$ of PPV-R were constructed in the pICPPV-SwCM backbone (Fig. 2D). As expected, all chimeras were able to infect $N$. clevelandii after mechanical inoculation of the corresponding DNA, so extracts from systemi-

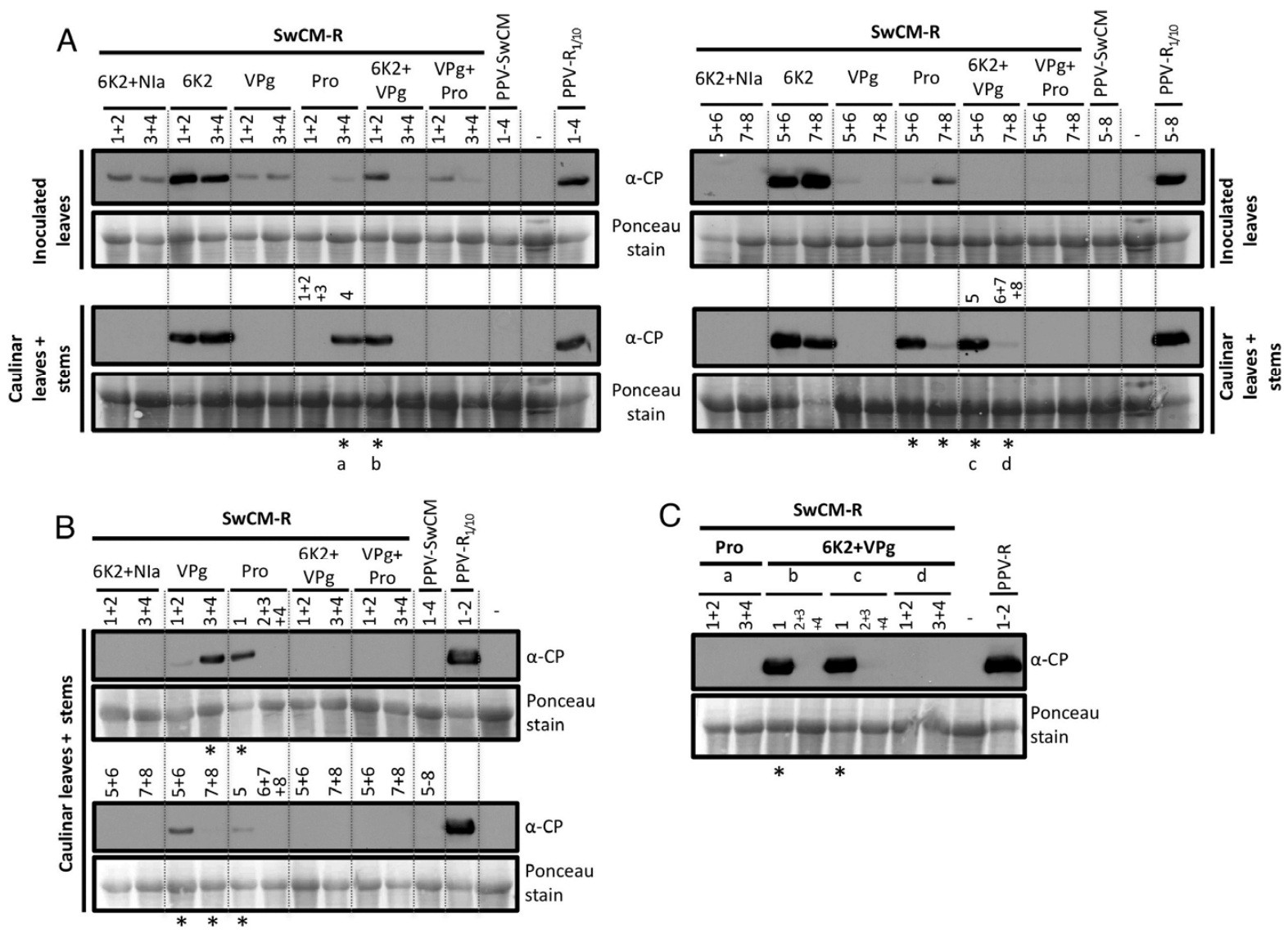

Fig. 4. Infectivity of chimeras derived from $6 \mathrm{~K} 2+\mathrm{NIaSwCM}-\mathrm{R}$ in Arabidopsis thaliana. $\mathbf{A}$ and $\mathbf{B}$, Viral accumulation assessed by anti-coat protein $(\mathrm{CP})$ immunoblot analysis (serum dilution 1:100,000) in inoculated rosette leaves and caulinar leaves plus stems collected at 9 and 21 days postinoculation (dpi), respectively, of plants of A. thaliana challenged with extracts from Nicotiana clevelandii plants infected with Plum pox virus (PPV)-SwCM, PPV-R, and the chimeras 6K2+NIaSwCM-R, 6K2SwCM-R, VPgSwCM-R, ProSwCM-R, 6K2+VPgSwCM-R, and VPg+ProSwCM-R. Plants inoculated with chimerical viruses were analyzed in groups of two or three, except for those that showed evident symptoms of systemic infection, which were analyzed individually. Plants inoculated with either PPV-SwCM or PPV-R (inoculum was diluted to 1/10 in healthy extract), were analyzed in groups of four (in B, only two plants were inoculated with PPV-R and they were analyzed together). The viral progeny from samples labeled a to $\mathrm{d}$ below the lanes was used to reinoculate new A. thaliana plants. Each panel shows the result of an independent experiment. C, Propagation of the viral progeny from systemically infected A. thaliana plants that had been challenged with chimeras ProSwCM-R (a) and 6K2+VPgSwCM-R (b, c, and d) and with PPV-R. The viral accumulation, assessed by anti-CP immunoblot analysis (serum dilution 1:100,000) at 21 dpi in caulinar leaves plus stems is shown. Plants were analyzed in groups of two or three, except for those inoculated with chimerical viruses that showed evident symptoms of systemic infection, which were analyzed individually. A. thaliana healthy samples were used as negative controls (-). Asterisks below the lanes indicate samples that were used for immunocapture reverse transcription-polymerase chain reaction followed by sequencing of the exchanged regions. Membranes stained with Ponceau red showing the Rubisco are included as loading controls in the lower panels. 
cally infected leaves of $N$. clevelandii were used as inocula to test the infectivity of the viral progeny from these constructs in A. thaliana and C. foetidum.

Local viral accumulation was detected in samples from $A$. thaliana inoculated with chimeras $6 \mathrm{~K} 2+\mathrm{NIa}+\mathrm{NIbR}-\mathrm{SwCM}$ (6K2+NIa and NIb from PPV-R in PPV-SwCM) and $6 \mathrm{~K} 2+\mathrm{NIa}+\mathrm{CPR}-\mathrm{SwCM}(6 \mathrm{~K} 2+\mathrm{NIa}$ and $\mathrm{CP}$ from PPV-R in PPV-SwCM) and, to a lesser extent, CI+6K2+NIaR-SwCM (CI and 6K2+NIa from PPV-R in PPV-SwCM), but systemic viral accumulation in caulinar leaves and stems was only detected in some plants infected with the $6 \mathrm{~K} 2+\mathrm{NIa}+\mathrm{NIbR}$ SwCM and 6K2+NIa+CPR-SwCM chimeras (Fig.6A).
According to this result, the possibility existed that to move systemically in $A$. thaliana, the chimera containing the $6 \mathrm{~K} 2+$ $\mathrm{NIa}+\mathrm{NIb}$ sequence from PPV-R would need to introduce mutations in the $\mathrm{CP}$ sequence from PPV-SwCM, and the chimera containing the $6 \mathrm{~K} 2+\mathrm{NIa}+\mathrm{CP}$ sequence from PPV-R would need to introduce mutations in the PPV-SwCM NIb sequence. To test this hypothesis, these genome regions were amplified by IC-RT-PCR and were sequenced. However, no mutations were found in the $\mathrm{CP}$ and $\mathrm{NIb}$ coding sequences of the different virus progenies.

The infectivity of the viral progeny from the chimeras combining P1-HCpro, P3+6K1, CI, NIb, or CP with $6 \mathrm{~K} 2+\mathrm{NIa}$ of

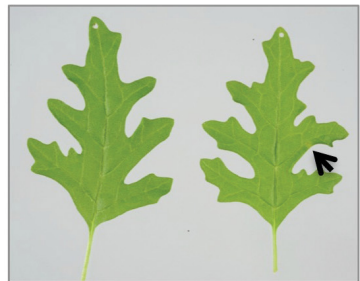

6K2+NlaSwCM-R

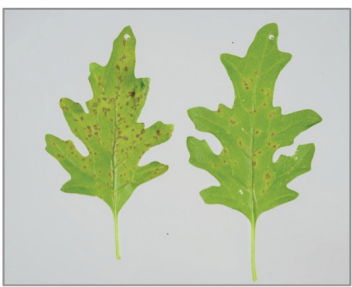

PPV-R

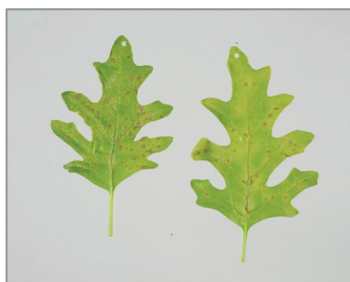

6K2SWCM-R

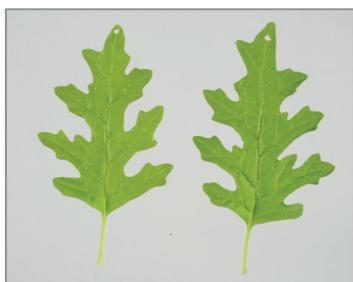

VPgSwCM-R

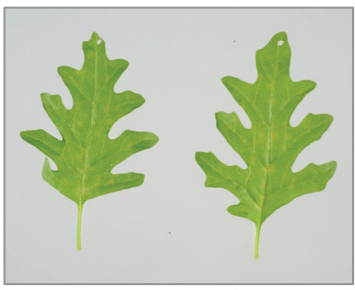

ProSwCM-R

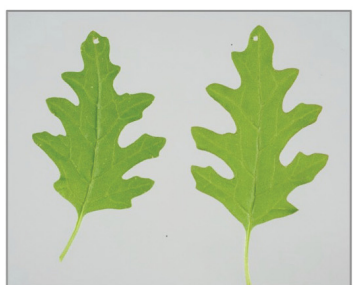

6K2+VPgSwCM-R

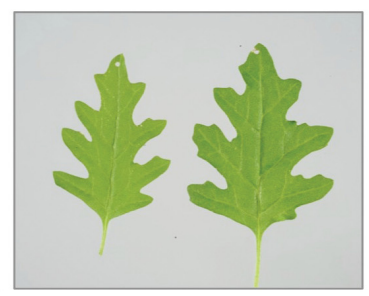

VPg+Pro SwCM-R

Fig. 5. Chenopodium foetidum leaves inoculated with the chimeras $6 \mathrm{~K} 2+\mathrm{NIaSwCM}-\mathrm{R}, 6 \mathrm{~K} 2 \mathrm{SwCM}-\mathrm{R}, \mathrm{VPgSwCM}-\mathrm{R}$, ProSwCM-R photographed at 8 days postinoculation. An isolated local lesion on one of the leaves inoculated with $6 \mathrm{~K} 2+\mathrm{NIaSwCM}-\mathrm{R}$ is indicated with a black arrow.

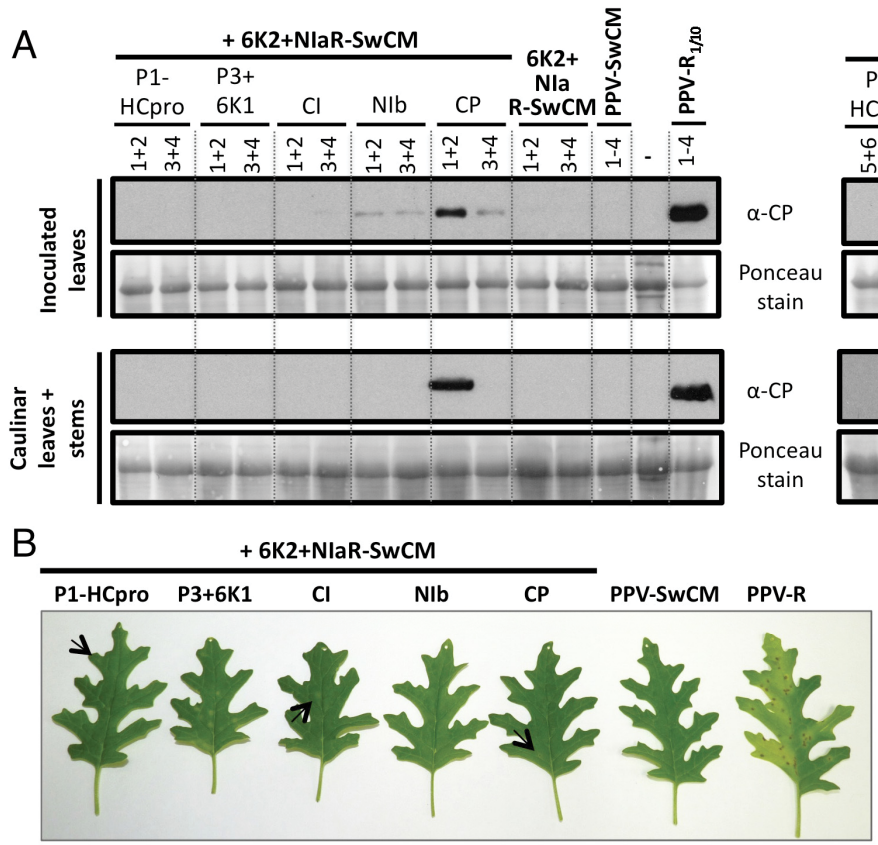

Fig. 6. Infectivity of chimeras derived from $6 \mathrm{~K} 2+\mathrm{NIaR}-\mathrm{SwCM}$. A, Viral accumulation assessed by anti-coat protein $(\mathrm{CP})$ immunoblot analysis (serum dilution 1:100,000) in inoculated rosette leaves and caulinar leaves plus stems collected at 9 and 21 days postinoculation (dpi), respectively, of Arabidopsis thaliana plants challenged with chimeras that combine the P1HCpro, P3+6K1, CI, NIb, and CP coding sequences of Plum pox virus (PPV)-R with those of $6 \mathrm{~K} 2+\mathrm{NIa}$ of the same virus in the pICPPV-SwCM backbone. Plants inoculated with each chimera were analyzed in pairs. A pool of four plants inoculated with PPV-R was used as a positive control. A sample from healthy A. thaliana (-) and a pool of four plants inoculated with PPV-SwCM were used as negative controls. Membranes stained with Ponceau red showing the Rubisco are included as loading controls in the lower panels. B, Pictures taken at 8 dpi of Chenopodium foetidum leaves inoculated with PPV-SwCM and PPV-R and the chimeras analyzed in the immunoblot analysis shown in A. Some isolated local lesions are indicated with black arrows. 
PPV-R in the pICPPV-SwCM backbone (Fig. 2D) was also tested in C. foetidum. In this host, scarce local lesions were observed on leaves inoculated with $6 \mathrm{~K} 2+\mathrm{NIaR}-, \mathrm{P} 1 \mathrm{HCpro}+6 \mathrm{~K} 2+$ NIaR-, CI+6K2+NIaR-, and 6K2+NIa+CPR-SwCM (Fig. 6B; Table 3 ). They were phenotypically similar to those caused by PPV-R. Interestingly, local lesions appeared to be more numerous than those observed on leaves inoculated with the aforementioned chimeras, although phenotypically milder than those produced by PPV-R at 8 dpi on leaves inoculated with the $\mathrm{P} 3+6 \mathrm{~K} 1+6 \mathrm{~K} 2+\mathrm{NIaR}-\mathrm{SwCM}$ chimera $(\mathrm{P} 3+6 \mathrm{~K} 1$ and $6 \mathrm{~K} 2+$ NIa from PPV-R in PPV-SwCM) (Fig. 6B; Table 3). Thus, a gain of infectivity as compared with $6 \mathrm{~K} 2+\mathrm{NIaR}-\mathrm{SwCM}$ was observed in this experiment.

According to these results, it seems that the $6 \mathrm{~K} 2+\mathrm{NIa}$ region of PPV-R is enough for PPV-SwCM to infect, although very inefficiently, C. foetidum. However, other viral regions, different for each host, are required to gain significant infectivity in C. foetidum (P3+6K1) and A. thaliana (either NIb or $\mathrm{CP}$ ).

\section{DISCUSSION}

All the major PPV strains are able to infect $C$. foetidum and A. thaliana, but this is not the case with PPV-C. Making use of chimeric cDNA clones, we have characterized genetic determinants in the PPV-SwCM sequence that render the virus noninfectious in A. thaliana and $C$. foetidum, and we have identified specific mutations involved in resistance breakdown. Our results highlight the importance of cooperative interactions between different viral products and host factors (most likely translation initiation factors) that are necessary for the establishment of an efficient viral infection.

\section{PPV-SwCM does not seem to trigger an $\boldsymbol{R}$ gene-mediated resistance mechanism in A. thaliana or C. foetidum.}

A previous report pointed to an $R$ gene-mediated resistance as the mechanism responsible for a first level of local defense preventing the infection of the PPV-SoC isolate (C strain) in $A$. thaliana (Decroocq et al. 2006). The negative result upon inoculation of PPV-SwCM in A. thaliana and C. foetidum suggests that either an ER response was triggered against PPV-SwCM or the lack of an essential host factor was preventing virus replication. However, the fact that no PPV-SwCM infection was produced in the A. thaliana rarl-10, and sgt 1 b-1/ rarl-10 mutants, which are defective in $R$ gene-mediated resistance, argues against the possibility that an ER mechanism triggered as a result of an $R$ gene-avr factor interaction is conferring resistance to these hosts.

If the avirulent isolate PPV-SwCM were triggering an ER response in A. thaliana or $C$. foetidum, small levels of replication that would have fallen under detection limits could have initiated a signaling cascade that would have inhibited the infection of PPV-R in the initially PPV-SwCM-inoculated leaves (local acquired resistance) or in upper leaves (systemic acquired resistance [SAR]). On the contrary, in co-inoculation experiments, PPV-R infection took place regardless of previous or simultaneous PPV-SwCM inoculation. We cannot rule out the possibility that PPV-SwCM is inducing local ER and SAR, but they are temporally uncoupled with the infection of the co-inoculated PPV-R. However, it seems more likely that resistance to PPV-SwCM is not mediated by an $R$ gene-related mechanism.

In case the resistance to PPV-SwCM was due to the lack of a compatible host-susceptibility factor, the reason why this deficiency could not be corrected in A. thaliana by co-inoculation with PPV-R could be that this host factor is only able to work in cis or because co-delivery of both viral constructs into the same cell occurred with a too-low frequency, as it has been previously reported (Zwart et al. 2011).

\section{The reciprocal exchange \\ of the 6K2+NIa coding sequences of PPV-R and \\ PPV-SwCM does not have a reciprocal effect.}

Chimerical clones between PPV-R and PPV-SwCM were constructed (Fig. 2A and B) in order to determine if the reciprocal exchange of any viral region could cause a gain of infectivity in PPV-SwCM or a decrease of infectivity in PPV-R in A. thaliana and C. foetidum.

All chimeras were infectious in $N$. clevelandii indicating that, in spite of the quite high divergence between the PPV-R and $\mathrm{C}$ strains $(78.6 \%$ nucleotide and $88.5 \%$ amino acid identities), their products are compatible in a fully susceptible host.

The results show that genetic determinants in the $6 \mathrm{~K} 2+\mathrm{NIa}$ region of PPV-SwCM are not compatible with virus infection in the restrictive hosts $A$. thaliana and C. foetidum. In contrast, the replacement of the $6 \mathrm{~K} 2+\mathrm{NI}$ a coding region of PPV-R by that of PPV-SwCM (chimera 6K2+NIaR-SwCM) did not cause a gain of infectivity (Table 2), which indicates that $6 \mathrm{~K} 2+$ NIa is not the only PPV-SwCM region that can be incompatible with $A$. thaliana and $C$. foetidum infection. Since the rest of chimeras based on PPV-R efficiently infected these hosts, the detrimental impact of PPV-SwCM sequences outside the $6 \mathrm{~K} 2+\mathrm{NIa}$ region depended on concurrent effects of more than one of the exchanged fragments.

\section{Mutations P114S (P1968S) and F163L (F2017L) \\ in VPg are associated with systemic infectivity of SwCM VPg-containing PPV chimeras in A. thaliana.}

PPV-R-based chimeras containing SwCM VPg or Pro separately (VPgSwCM-R and ProSwCM-R) were able to systemically infect $A$. thaliana, although not all inoculated plants (Fig. $4 \mathrm{~A}$ and $\mathrm{B}$ ). While the viral progeny of ProSwCM-R did not display mutations in the exchanged region (Pro), two independent mutations, P114S and F163L (amino acid positions relative to the VPg protein; which correspond to P1968S and F2017L in the full-length genomic polyprotein) were introduced in the SwCM VPg of $6 \mathrm{~K} 2+\mathrm{VPgSwCM}-\mathrm{R}$ and VPgSwCM-R (Table 4). Simultaneous presence of VPg and Pro from SwCM made the chimera $\mathrm{VPg}+\mathrm{ProSwCM}-\mathrm{R}$ not infectious in any experiment.

Recessive resistance breakdown associated with mutations in the VPg coding sequences have frequently been reported in many plant-potyvirus systems. Single amino acid changes in the VPg are enough to overcome resistance mechanisms based on eIF4E/eIF(iso)4E-VPg interactions. For instance, five independent mutations in the region between amino acids 101 and 120 of VPg were found to confer virulence to the Potato virus $Y$ (PVY) isolate SON41p in pepper (Ayme et al. 2006), two different amino acids, $\mathrm{L}$ and $\mathrm{H}$, at position 113 of VPg conferred virulence to Tobacco etch virus (TEV) in two resistant pepper genotypes with different eIF4E alleles (Perez et al. 2012) and two independent mutations in VPg, E116Q and N163Y, restored Turnip mosaic virus (TuMV) virulence in knockout A. thaliana mutants defective in eIF(iso)4E and eIF(iso)4G (Gallois et al. 2010) (Fig. 7). Moreover, contribution of small changes in VPg to adaptation to new hosts, probably without involving eIF4E, has also been reported (Svanella-Dumas et al. 2014).

Both PPV-SwCM VPg mutations, P114S and F163L, that were introduced in the $6 \mathrm{~K} 2+\mathrm{VPgSwCM}-\mathrm{R}$ and $\mathrm{VPgSwCM}-\mathrm{R}$ chimeras are located in the central and C-terminal regions of $\mathrm{VPg}$, which are the regions that are supposed to interact with the eIF4E factor. Proline 114 of PPV-SwCM VPg is replaced by methionine in PPV-R (Fig. 7) and in most PPV isolates that do not belong to strains $\mathrm{C}$ and CR. Amino acid 114 of PPV 
VPg aligns with amino acids 115 and 113 of the VPg proteins of PVY and TEV, respectively, which are involved in resistance breaking. Moreover, mutation F163L of PPV VPg is coincident with that of a resistance-breaking TuMV mutant. In contrast to the PPV-SwCM VPg mutation P114S, the F163L mutation is in a quite conserved protein region (Fig. 7; Supplementary Fig. S2).

Thus, these results strongly suggest that the resistance breakdown of chimeras $6 \mathrm{~K} 2+\mathrm{VPgSwCM}-\mathrm{R}$ and $\mathrm{VPgSwCM}-\mathrm{R}$ requires the introduction of mutations in VPg regions that restore functional interactions with the translation initiation machinery of A. thaliana. Although further experiments are needed, this appears to indicate that, whereas the VPg of PPV-D isolates is able to interact functionally with eIF4E or eIF(iso)4E isoforms of both Nicotiana spp. and A. thaliana, the VPg of PPV-C isolates is only able to do it with an eIF4E/eIF(iso)4E factor of Nicotiana spp. On the other hand, our results also suggest that other viral factors participate in this interaction and condition infectivity, since VPg mutations breaking resistance have only appeared in chimerical clones and never in wild-type PPV-SwCM. This is in agreement with the fact that not only mutations in VPg but also in other viral proteins have been found to be able to break eIF4E-mediated resistance (Abdul-Razzak et al. 2009; Nakahara et al. 2010), and physical interaction between HCpro and eIF4E/eIF(iso)4E has been demonstrated (Ala-Poikela et al. 2011).

\section{Both VPg and Pro domains of NIa could be involved in the putative interaction with the host factor limiting PPV-C infection in A. thaliana and C. foetidum.}

Whereas the chimera VPgSwCM-R containing VPg from PPV-SwCM was infectious in some A. thaliana plants, introducing mutations in the $\mathrm{VPg}$ sequence $\mathrm{VPg}+\mathrm{ProSwCM}-\mathrm{R}$, which contains the complete NIa (VPg-Pro) from the avirulent virus, was never found to be infectious in this host (Table 4). These results could suggest that, although the NIa protease domain of PPV-SwCM is not completely incompatible with the infection of $A$. thaliana, this protein region is also relevant for the putative interaction with the host factor that conditions PPV infectivity in this host.

Thus, this interaction could be established by the VPg domain of the entire NIa (VPg+Pro) rather than by free VPg. In fact, it has been reported that eIF4E isoforms can interact with mature VPg or the VPg domain of partially processed precursors (Jiang and Laliberté 2011). More precisely, in TuMV, the mature VPg has not been detected in infected cells, and its precursor NIa (VPg+Pro) appears to be the VPg-related factor that associates with the eIF4(iso)4E in vivo (Beauchemin et al. 2007). Thus, the entire NIa seems to be important for eIF4E/ eIF(iso)4E-VPg interactions.

In C. foetidum, chimera ProSwCM-R caused delayed mild lesions, as compared with those provoked by PPV-R (Fig. 5), which eventually developed necrosis, thus indicating that the NIapro domain plays a role, albeit secondary, in the mechanism that prevents PPV-SwCM infection in $C$. foetidum, similarly as in A. thaliana.

\section{Different viral factors, in addition to NIa, contribute to the inability of PPV-SwCM to infect $A$. thaliana and C. foetidum.}

As mentioned above, single mutations in VPg have been shown to allow avirulent virus isolates to infect resistant genotypes of susceptible plant species (Ayme et al. 2006; Gallois et al. 2010; Perez et al. 2012). Adaptation of PPV-C isolates to resistant host species, $A$. thaliana and $C$. foetidum, requires more complex genome rearrangements, with the concurrence of changes in VPg and other viral products.

Chimeras based on $6 \mathrm{~K} 2+\mathrm{NIaR}-\mathrm{SwCM}$, in which additional PPV-R coding sequences replaced those of PPV-SwCM, provide important clues on this matter (Fig. 2D). Chimeras systemically infecting $A$. thaliana $(6 \mathrm{~K} 2+\mathrm{NIa}+\mathrm{NIbR}-$ and $6 \mathrm{~K} 2+$ $\mathrm{NIa}+\mathrm{CPR}-\mathrm{SwCM}$ ) were different from the chimera causing local lesions in $C$. foetidum (P3+6K1+6K2+NIaR-SwCM). These combinations conferred a gain of competence but not to the levels of PPV-R (Fig. 6A and B). Thus, although NIb or CP (for A. thaliana) and $\mathrm{P} 3+6 \mathrm{~K} 1$ (for $C$. foetidum) of PPV-R

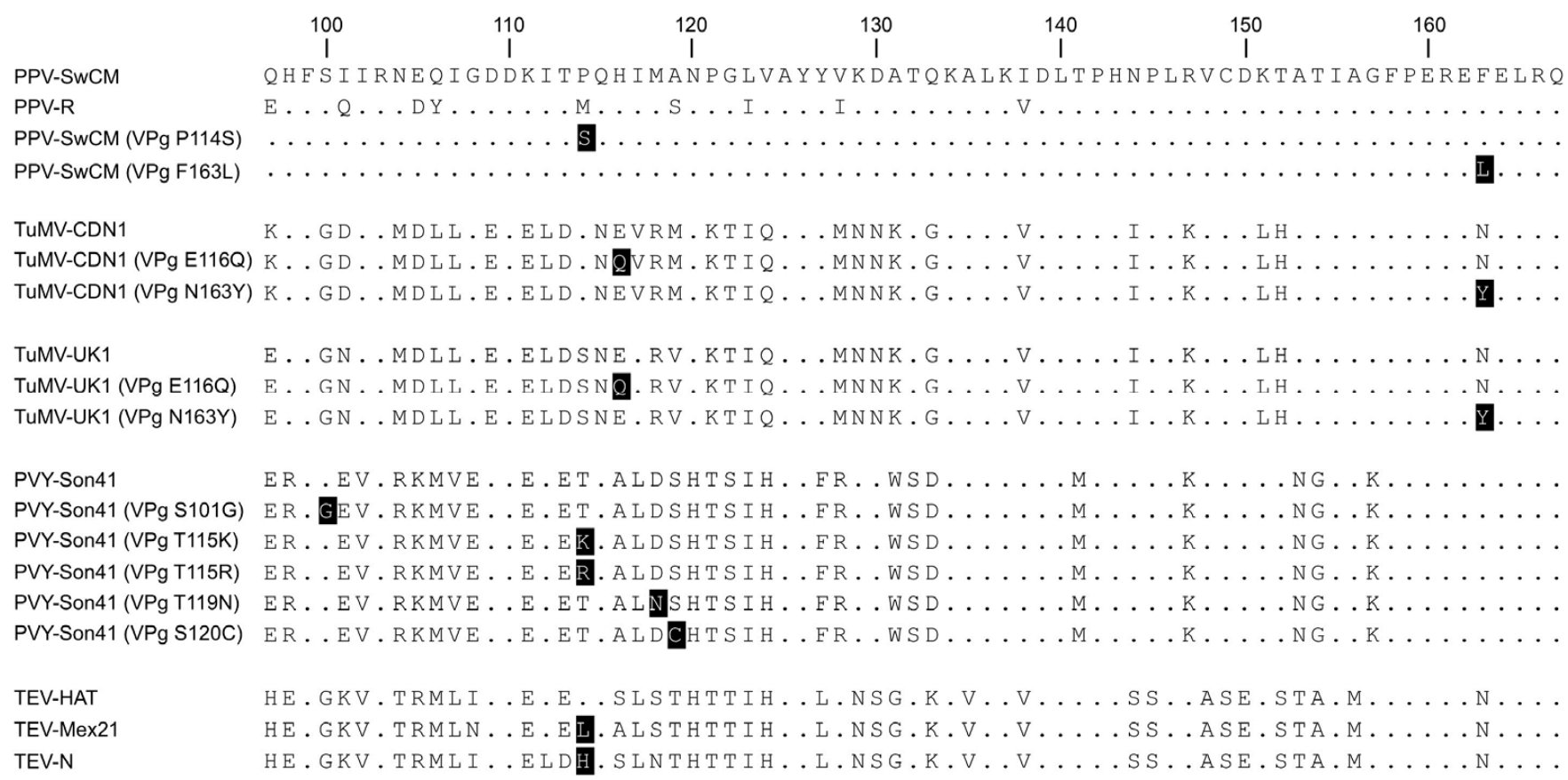

Fig. 7. Alignment of genome-linked viral protein (VPg) sequences from potyvirus isolates, including mutations that enable breakdown of eIF4E/eIF(iso)4Ederived resistance (shown on a black background). Amino acids identical to those of PPV-SwCM are indicated by dots. PPV, Plum pox virus; TuMV, Turnip mosaic virus; PVY, Potato virus Y; and TEV, Tobacco etch virus. 
increased the infectivity of chimeras also containing $6 \mathrm{~K} 2+\mathrm{NIa}$ of PPV-R, other viral regions seem to be needed to reach an optimal competence. This is expected, given that genetic determinants involved in host adaptation can be spread through the entire viral genome (Salvador et al. 2008).

The different combinations that confer a gain of infectivity in $A$. thaliana and $C$. foetidum could suggest the establishment of species-specific multiprotein interactions in which NIa (VPg+Pro) seems to be a core component, since the single exchange of $\mathrm{P} 3+6 \mathrm{~K} 1, \mathrm{NIb}$, or $\mathrm{CP}$ had no effect on the infectivity of the chimeras, as compared with the parental viruses. The disordered nature of VPg enables its interaction with many other proteins, not only from the host but, also, from the virus. It has been shown that $6 \mathrm{~K} 2+\mathrm{NIa}$ is, indeed, able to interact with $\mathrm{P} 3$, NIb, and $\mathrm{CP}$, among other proteins, as well as with viral RNA in viral replication complexes (Jiang and Laliberté 2011). In the case of PPV, bimolecular fluorescence complementation assays have revealed that the NIa protease domain interacts with all viral proteins except for P1 and HCpro, while the VPg domain interacts with all except for P1, HCpro, P3, and 6K1 (Zilian and Maiss 2011). Moreover, the in vitro interaction of NIapro and NIb has been reported for TEV, and it is supposed to be necessary for TEV replication (Daròs et al. 1999). Thus, it seems that P3+6K1, NIb, or CP could participate in specific host-virus interactions nucleated by NIa by binding the NIapro domain.

\section{A resistance mechanism based on the lack of an eIF4E/eIF(iso) $4 \mathrm{E}-\mathrm{VPg}$ interaction might prevent PPV-SwCM infection in A. thaliana and $C$. foetidum.}

All together, the results reported here suggest that the mechanism of resistance to PPV-C in the restrictive hosts is mainly triggered by the incompatibility of $\mathrm{NIa}(\mathrm{VPg}+\mathrm{Pro})$ with specific host factors. Mutations in the VPg coding sequence can cause resistance breakdown, but additional factors that may be located along the entire genome (especially those probably involved in the viral replication complex), also participate. The position of the mutations detected in the VPg sequence strongly suggests that the host factor involved in this interaction could be a component of the translation initiation machinery (an eIF4E isoform), although further experiments are needed to confirm this hypothesis. The results of this work strongly suggest that translation initiation factors might, in addition to defining the most common mechanism for passive resistance of particular genotypes of host plants against potyviruses (Robaglia and Caranta 2006; Truniger and Aranda 2009; Wang and Krishnaswamy 2012), also contribute to establishment of nonhost resistance and, thus, to delimit potyvirus host-ranges. A recent report about overcoming nonhost resistance to TEV by complementation with eIF4E from a host species (Estevan et al. 2014) further supports this conclusion. The crucial role of translation initiation factors in delimiting plant-virus compatibility appears to extend beyond the potyvirus world, as nonhost resistance of $N$. benthamiana to a carmovirus has been shown to result from an incompatible interaction between virus RNA and eIF4E (Nieto et al. 2011). Moreover, our results argue against simple genefor-gene interactions governing resistance mediated by translation initiation factors and underscore the complex interrelationships between viral and host factors to launch a successful viral infection.

\section{MATERIALS AND METHODS}

\section{Plant hosts.}

Nicotiana clevelandii and Chenopodium foetidum were maintained under greenhouse conditions (16 h of light with supplementary illumination when needed and a temperature range of 19 to $23^{\circ} \mathrm{C}$ ). Arabidopsis thaliana seeds were germinated and maintained in a growth chamber (8-h-light photoperiod and a temperature of $22^{\circ} \mathrm{C}$ ).

A. thaliana rarl-10 and sgtlb-1/rarl-10 mutants (Austin et al. 2002) were provided by V. Decroocq (INRA, France).

\section{Construction of full-length cDNA infectious clones.}

Full-length cDNA clones derived from PPV isolates PPV-R and PPV-SwCMp were constructed as described by LópezMoya and García (2000) and Calvo and associates (2014), respectively. The construction of chimerical clones is described in Supplementary Text S1. PCR reactions were performed with Pwo DNA polymerase (Roche Applied Science, Indianapolis, IN, U.S.A.), using the specific primers listed in Supplementary Table S1. DNA digests were carried out using either New England Biolabs (Ipswich, MA, U.S.A.) or Thermo Fisher Scientific (Waltham, MA, U.S.A.) restriction enzymes. All ligations were performed using T4 DNA ligase (Thermo Fisher Scientific). Ligation mixtures were transformed into E. coli DH10b or DH5 $\alpha$ either by electroporation or heat-shock.

\section{Mechanical hand inoculation.}

For the inoculation of pICPPV-R, pICPPV-SwCM, and their derived chimerical clones, plasmid minipreps were prepared at a concentration of approximately $250 \mathrm{ng} / \mu \mathrm{l}$ and approximately $25 \mu \mathrm{l}$ of DNA per plant was distributed and directly rubbed on three leaves of $N$. clevelandii.

For manual inoculation of the viral progeny obtained from infected plants, systemically infected leaves were ground to a fine powder under liquid nitrogen and were homogenized with $5 \mathrm{mM}$ sodium phosphate buffer, $\mathrm{pH} 7.4$ (2 $\mathrm{ml}$ per gram of tissue) using vortex. The extracts (15 to $20 \mu \mathrm{l})$ were used to inoculate six leaves of A. thaliana, two to four leaves of $C$. foetidum, or three leaves of $N$. clevelandii. All plants were dusted with Carborundum prior to inoculation.

\section{Immunoblot analysis.}

Leaves were collected and ground to a fine powder under liquid nitrogen and were stored at $-80^{\circ} \mathrm{C}$ until use. Protein extracts were prepared by thawing the powder in extraction buffer (150 mM Tris- $\mathrm{HCl}, \mathrm{pH} 7.5,6 \mathrm{M}$ urea, 2\% sodium dodecyl sulphate (SDS), and 5\% $\beta$-mercaptoethanol) ( $2 \mathrm{ml}$ per gram of tissue). Samples were boiled for $10 \mathrm{~min}$, and cell debris was removed by centrifugation at $18,000 \times g$ at $4^{\circ} \mathrm{C}$ for 10 min. Supernatants were resolved on SDS-polyacrylamide gels (12\% acrylamide) and were electroblotted to a nitrocellulose membrane. Ponceau red staining was used to check the global protein content of samples. In house-prepared anti-PPV CP rabbit serum was used as primary antibody and horseradish peroxidase-conjugated goat antirabbit immunoglobulin G (Jackson ImmunoResearch, West Grove, PA, U.S.A) as secondary reagent. The immunostained proteins were visualized by enhanced chemiluminescence detection with a LiteABlot kit (Euroclone S.p.A., Siziano, Italy).

\section{IC-RT-PCR.}

For IC-RT-PCR assays, A. thaliana samples were homogenized in $5 \mathrm{mM}$ sodium phosphate buffer, $\mathrm{pH} 7.4$ (2 ml per gram of tissue), using vortex, and was centrifuged at $18,000 \times g$ at $4^{\circ} \mathrm{C}$ for $2 \mathrm{~min}$. The supernatant was incubated overnight at $4^{\circ} \mathrm{C}$ in tubes previously coated with anti-PPV immunoglobulins (Steinbuch and Audran 1969). The incubation was followed by three washing steps with PBS-Tween buffer $(16 \mathrm{mM}$ sodium phosphate buffer, $0.1 \mathrm{M} \mathrm{NaCl}, 0.05 \%$ Tween 20, $\mathrm{pH}$ 7.2). RTPCR tests were performed using a Titan kit (Roche Molecular Biochemicals, Branchburg, NJ, U.S.A.) with specific primers targeting the sequences to be amplified. For sequence analysis, 
RT-PCR products were purified using the FavorPrep Gel/PCR purification mini kit (Favorgen Biotech. Corp., Ping-Tung, Taiwan) and sequenced by Macrogen Europe (Amsterdam).

\section{ACKNOWLEDGMENTS}

We thank B. García for technical assistance and D. San León for help in bioinformatics analyses. This work was supported by grants BIO201018541 and BIO2013-49053-R from the Spanish government, and KBBE204429 from the European Union. M. Calvo was the recipient of an I3P fellowship from Consejo Superior de Investigaciones Científicas-Fondo Social Europeo. M. Calvo performed all the experiments shown in this article, and participated in the experimental design and in writing the manuscript. S. Martínez-Turiño performed control experiments essential to validate some of the results, and critically revised the intellectual content of the article. J. A. García coordinated the work and participated in the experimental design and in writing the manuscript. All authors read and approved the final manuscript.

\section{LITERATURE CITED}

Abdul-Razzak, A., Guiraud, T., Peypelut, M., Walter, J., Houvenaghel, M. C., Candresse, T., Gall, O., and German-Retana, S. 2009. Involvement of the cylindrical inclusion (CI) protein in the overcoming of an eIF4Emediated resistance against Lettuce mosaic potyvirus. Mol. Plant Pathol. 10:109-113.

Ala-Poikela, M., Goytia, E., Haikonen, T., Rajamaki, M.-L., and Valkonen, J. P. T. 2011. Helper component proteinase of genus Potyvirus is an interaction partner of translation initiation factors eIF(iso)4E and eIF4E that contains a 4E binding motif. J. Virol. 85:6784-6794.

Austin, M. J., Muskett, P., Kahn, K., Feys, B. J., Jones, J. D., and Parker, J. E. 2002. Regulatory role of SGT1 in early $R$ gene-mediated plant defenses. Science 295:2077-2080.

Ayme, V., Souche, S., Caranta, C., Jacquemond, M., Chadoeuf, J., Palloix, A., and Moury, B. 2006. Different mutations in the genome-linked protein VPg of Potato virus $Y$ confer virulence on the $p v r 2^{3}$ resistance in pepper. Mol. Plant Microbe Interact. 19:557-563.

Azevedo, C., Sadanandom, A., Kitagawa, K., Freialdenhoven, A., Shirasu, K., and Schulze-Lefert, P. 2002. The RAR1 interactor SGT1, an essential component of $R$ gene-triggered disease resistance. Science 295:2073-2076.

Beauchemin, C., Boutet, N., and Laliberté, J. F. 2007. Visualization of the interaction between the precursors of VPg, the viral protein linked to the genome of Turnip mosaic virus, and the translation eukaryotic initiation factor iso 4E in planta. J. Virol. 81:775-782.

Calvo, M., Malinowski, T., and García, J. A. 2014. Single amino acid changes in the 6K1-CI region can promote the alternative adaptation of Prunus- and Nicotiana-propagated Plum pox virus $\mathrm{C}$ isolates to either gost. Mol. Plant Microbe Interact. 27:136-149.

Cooper, B. 2001. Collateral gene expression changes induced by distinct plant viruses during the hypersensitive resistance reaction in Chenopodium amaranticolor. Plant J. 26:339-349.

Crescenzi, A., d'Aquino, L., Comes, S., Nuzzaci, M., Piazzolla, P., Boscia, D., and Hadidi, A. 1997. Characterization of the sweet cherry isolate of plum pox potyvirus. Plant Dis. 81:711-714.

Daròs, J. A., Schaad, M. C., and Carrington, J. C. 1999. Functional analysis of the interaction between VPg-proteinase (NIa) and RNA polymerase (NIb) of tobacco etch potyvirus, using conditional and suppressor mutants. J. Virol. 73:8732-8740.

Decroocq, V., Sicard, O., Alamillo, J. M., Lansac, M., Eyquard, J. P., García, J. A., Candresse, T., Le Gall, O., and Revers, F. 2006. Multiple resistance traits control Plum pox virus infection in Arabidopsis thaliana. Mol. Plant Microbe Interact. 19:541-549.

Elena, S. F., Carrera, J., and Rodrigo, G. 2011. A systems biology approach to the evolution of plant-virus interactions. Curr. Opin. Plant Biol. 14:372-377.

Estevan, J., Marena, A., Callot, C., Lacombe, S., Moretti, A., Caranta, C., and Gallois, J. L. 2014. Specific requirement for translation initiation factor $4 \mathrm{E}$ or its isoform drives plant host susceptibility to Tobacco etch virus. BMC Plant Biol 14:67.

Fanigliulo, A., Comes, S., Maiss, E., Piazzolla, P., and Crescenzi, A. 2003. The complete nucleotide sequence of Plum pox virus isolates from sweet (PPV-SwC) and sour (PPV-SoC) cherry and their taxonomic relationships within the species. Arch. Virol. 148:2137-2153.

Gallois, J. L., Charron, C., Sanchez, F., Pagny, G., Houvenaghel, M. C., Moretti, A., Ponz, F., Revers, F., Caranta, C., and German-Retana, S.
2010. Single amino acid changes in the turnip mosaic virus viral genome-linked protein (VPg) confer virulence towards Arabidopsis thaliana mutants knocked out for eukaryotic initiation factors eIF(iso)4E and eIF(iso)4G. J. Gen. Virol. 91:288-293.

García, J. A., Glasa, M., Cambra, M., and Candresse, T. 2014. Plum pox virus and sharka: A model potyvirus and a major disease. Mol. Plant Pathol. 15:226-241.

Goodin, M. M., Zaitlin, D., Naidu, R. A., and Lommel, S. A. 2008. Nicotiana benthamiana: Its history and future as a model for plant-pathogen interactions. Mol. Plant Microbe Interact. 21:1015-1026.

Ivanov, K. I., Eskelin, K., Lõhmus, A., and Mäkinen, K. 2014. Molecular and cellular mechanisms underlying potyvirus infection. J. Gen. Virol. 95:1415-1429.

Jiang, J., and Laliberté, J. F. 2011. The genome-linked protein VPg of plant viruses-a protein with many partners. Curr. Opin. Virol. 1:347-354.

Llácer, G. 2006. Hosts and symptoms of Plum pox virus: Herbaceous hosts. EPPO Bulletin 36:227-228.

López-Moya, J. J., and García, J. A. 2000. Construction of a stable and highly infectious intron-containing cDNA clone of plum pox potyvirus and its use to infect plants by particle bombardment. Virus Res. 68:99107.

López-Moya, J. J., Valli, A., and García, J. A. 2009. Potyviridae. Encyclopedia of Life Sciences (ELS). John Wiley \& Sons, Ltd: Chichester [DOI: 10.1002/9780470015902.a9780470000755.pub9780470015902].

Moffett, P. 2009. Mechanisms of recognition in dominant $R$ gene mediated resistance. Adv. Virus Res. 75:1-33.

Nakahara, K. S., Shimada, R., Choi, S.-H., Yamamoto, H., Shao, J., and Uyeda, I. 2010. Involvement of the P1 cistron in overcoming eIF4Emediated recessive resistance against Clover yellow vein virus in pea. Mol. Plant Microbe Interact. 23:1460-1469.

Nieto, C., Rodríguez-Moreno, L., Rodríguez-Hernández, A. M., Aranda, M. A., and Truniger, V. 2011. Nicotiana benthamiana resistance to nonadapted Melon necrotic spot virus results from an incompatible interaction between virus RNA and translation initiation factor 4E. Plant J. 66:492-501.

Ouibrahim, L., and Caranta, C. 2013. Exploitation of natural genetic diversity to study plant-virus interactions: What can we learn from Arabidopsis thaliana? Mol. Plant Pathol. 14:844-854.

Perez, K., Yeam, I., Kang, B. C., Ripoll, D. R., Kim, J., Murphy, J. F., and Jahn, M. M. 2012. Tobacco etch virus infectivity in Capsicum Spp. is determined by a maximum of three amino acids in the viral virulence determinant VPg. Mol. Plant Microbe Interact. 25:1562-1573.

Robaglia, C., and Caranta, C. 2006. Translation initiation factors: A weak link in plant RNA virus infection. Trends Plant Sci 11:40-45.

Salvador, B., Delgadillo, M. O., Saénz, P., García, J. A., and Simón-Mateo, C. 2008. Identification of Plum pox virus pathogenicity determinants in herbaceous and woody hosts. Mol. Plant Microbe Interact. 21:20-29.

Steinbuch, M., and Audran, R. 1969. The isolation of IgG from mammalian sera with the aid of caprylic acid. Arch. Biochem. Biophys 134:279-284.

Šubr, Z., and Glasa, M. 2013. Unfolding the secrets of plum pox virus: From epidemiology to genomics. Acta Virol. 57:217-228.

Sutic, D., Jordovic, M., Rankovic, M., and Festic, H. 1971. Comparative studies of some sharka (plum pox) virus isolates. Ann. Phytopathol. Proceedings of the VIII Symposium sur les Maladies a Virus des Arbres Frutiers:185-192.

Svanella-Dumas, L., Verdin, E., Faure, C., German-Retana, S., Gognalons, P., Danet, J. L., Marais, A., and Candresse, T. 2014. Adaptation of Lettuce mosaic virus to Catharanthus roseus involves mutations in the central domain of the VPg. Mol. Plant Microbe Interact. 27:491-497.

Truniger, V., and Aranda, M. A. 2009. Recessive resistance to plant viruses. Adv. Virus Res. 75:119-159.

van Dijk, P., van der Meer, F. A., and Piron, P. G. M. 1987. Accessions of Australian Nicotiana species suitable as indicator hosts in the diagnosis of plant virus diseases. Neth. J. Plant Pathol. 93:73-85.

Wang, A., and Krishnaswamy, S. 2012. Eukaryotic translation initiation factor 4E-mediated recessive resistance to plant viruses and its utility in crop improvement. Mol. Plant Pathol. 13:795-803.

Yongqiang, Z., Xinwu, P., Chao, Z., Zifeng, L., Zhixing, W., Shirong, J., and Weimin, L. 2012. De novo foliar transcriptome of Chenopodium amaranticolor and analysis of its gene expression during virus-induced hypersensitive response PLoS One 7:e45953. Published online.

Zilian, E., and Maiss, E. 2011. Detection of plum pox potyviral proteinprotein interactions in planta using an optimized mRFP-based bimolecular fluorescence complementation system. J. Gen. Virol. 92:27112723

Zwart, M. P., Daròs, J. A., and Elena, S. F. 2011. One is enough: In vivo effective population size is dose-dependent for a plant RNA virus. PLoS Pathog. 7:e1002122. Published online. 